\title{
Effect of Training Functions of Artificial Neural Networks (ANN) on Time Series Forecasting
}

\author{
Rashi Aggarwal \\ Jamia Millia Islamia University, \\ India
}

\author{
Rajendra Kumar, Ph.D. \\ Assistant Professor, \\ Department of Computer Science \\ Jamia Millia Islamia University, India
}

\begin{abstract}
Weather forecasting has been an area of considerable interest among researchers since long. A scientific approach to weather forecasting is highly dependent upon how well the atmosphere and its interactions with the various aspects of the earth surface is understood. Applicability of artificial neural networks (ANNs) in forecasting has led to tremendous surge in dealing with uncertainties. This paper focuses on analysis and selection of various techniques used in developing a suitable feed forward neural network for forecasting $24 \mathrm{hr}$ ahead hourly temperature using MATLAB 7.6.0 neural network toolbox. The data of 60 days hourly data temperature is used to train and test the different models, training functions, activation functions, learning functions, performance functions and the most suitable combination is selected. The performance and reliability of these models are then evaluated by number of statistical measures. Results are compared with each training function.
\end{abstract}

\section{INTRODUCTION}

The weather is a continuous, data-intensive, multidimensional, dynamic and chaotic process and these properties make weather forecasting a challenging and complicated procedure that includes multiple specialized fields of expertise. Generally two methods are used to forecast weather: (a) the empirical approach and (b) the dynamical approach ${ }^{11]}$. The first approach is useful when records are plentiful and the second involves differential equations which are often used to model large scale weather phenomena. However, little attention has been paid on the use of artificial neural networks in weather forecasting ${ }^{[2][3][4]}$.

With the advent in research activities, artificial neural networks have shown considerable results due to its powerful pattern classification and pattern recognition capabilities. ANNs are data-driven self-adaptive methods which learn from examples and capture subtle functional relationships even if the underlying relationships are unknown ${ }^{[5]}$. Atmospheric processes exhibit certain spatial and temporal variability and hence issues of non-linearity exist frequently. ANNs extract the relationship between input and output processes, without the physics being explicitly provided ${ }^{[6]}$.

Generalization ability of data variables also improved with the artificial neural networks, constructed in two steps: (i) training a given number of neural networks and then, (ii) combining the predictions. It can often correctly infer the unseen part of a population even if it contains noisy data ${ }^{[7]}$. The objective of this study is to develop in-depth understanding of various predictive models for forecasting $24 \mathrm{hr}$ ahead temperature and then comparing these models.

\section{PROPOSED WORK}

This section deals with collection of data sets having parameters as maximum and minimum temperature. The dataset is gathered from meteorological department. The use of original data as the input may cause convergence problem ${ }^{[8]}$ therefore data pre-processing operations are performed and data is normalized through min-max normalization technique to make it in a range of $[-1,1]$. At the end of each algorithm, the outputs are denormalized into the original data format to get the desired results ${ }^{[9]}$. The configuration of neural network architecture depends upon the problem. Thus trial and error method is adopted to choose the number of hidden layers. The architecture with 24 hidden neurons was faster in computation but the convergence rate was slow therefore 48 hidden neurons were chosen to balance computation and convergence.

\section{METHODOLOGY}

The approach used for analyzing different parameters in the MATLAB toolbox, is given by the following steps:

\subsection{Data Segmentation}

The weather parameters, including minimum and maximum temperature were collected from the database for analyzing the intelligent-based forecasting models. To examine the next day temperature, the data is split into three sets: train, validation and test sets. The train set is used to train the network data, and the validation set is used to examine the training performance. Further test set is used to evaluate the true accuracy of prediction. The method is applied on fully connected feedforward multilayer perceptron with various activation functions at hidden layer and linear activation function at output layer.

\subsection{Data Normalization}

Data normalization is a pre-processing strategy that transforms time series into specified range typically either between $[0,1]$ or $[-1,1]$. The quality of data is guaranteed when normalized data is fed to a learning algorithm. Of all the techniques available such as decimal scaling, Z- score, MinMax etc, Min-Max techniques is chosen for normalizing the data and making it in a range of $[0,1]$ which is defined as follows:

$V^{\prime}=(v-\min A)\left(\left(n e w \_\max A\right)+n e w \_\min A\right) / \quad(\max A-$ $\min A)$

Where $v^{\prime}$ is the normalized data, $v$ is the original data of attribute $\mathrm{A}$

$\max \mathrm{A}$ is the maximum value of attribute $\mathrm{A}$, mina is the minimum value of an attribute A. A min-max normalization maps a value $\mathrm{v}$ of attribute $\mathrm{A}$ to $\mathrm{v}$ ' in the range [ new_minA, new_maxA], new_minA is set to 0.0 and new_maxA to $1.0 .^{[10]}$ 


\subsection{Parameter Initialization}

Select the set of parameters to train the network. The main parameters selected are number of epochs, learning rate, performance goal with mean squared error(MSE) and minimum and maximum gradient.

\subsection{Training}

Train the network based on a set of activation functions and number of neurons. The number of neurons at the hidden layer are varied to get the optimum combination where training period and performance error is considerably less.

\subsection{Performance Evaluation for these Models}

To compare actual versus predicted temperature using the ANN model, various statistical parameters were taken into consideration: mean square error and computational speed.

Figure1 shows the proposed feed forward neural network. The architecture consists of an input layer, a hidden layer and an output layer. The input layer consists of 24 neurons at the input layer where each neuron contains the hourly data of each day, 48 neurons at the hidden layer and 24 neurons at the output layer, where each neuron gives the hourly temperature of next day as an output. To train the network, various combinations of training algorithms were taken to get the optimized architecture. Training data consisted of first 40 days and test data consisted of last 20 days. Pre and postprocessing functions are used to have more efficient training ${ }^{[11]}$. Various models have already been explored such as ANN and MLR, however ANN proved to exhibit certain non-linear relationship. Therefore ANN has been selected here. ${ }^{[12]}$

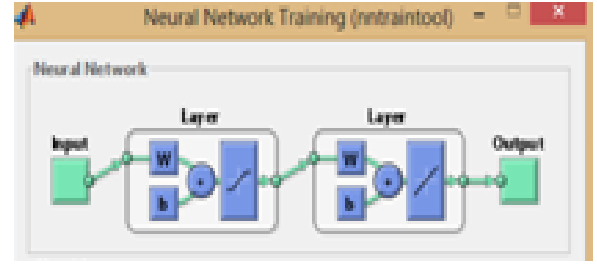

Figure 1: Proposed Network Architecture

\section{RESULTS AND DISCUSSION}

For the prediction of one day ahead each hour temperature, a feed forward neural network with back propagation algorithm was implemented. The input data were max and min temperature of 60 days. The network was trained using supervised learning by taking data for first 40 days. To test the accuracy of the network last 20 days data were introduced to the network.

Comparison of the training functions for the forecasting of temperature are shown in table 1 and analyzed in following section.

Figure 3 indicates that TRAINLM Incurred lesser errors and is capable of representing non-linear functions better than other training functions. To check the response of the network, regression analysis has been conducted between the target and the output. It was observed that 'TRAINLM' showed a correlation coefficient of 0.93 which is again better than other training functions.

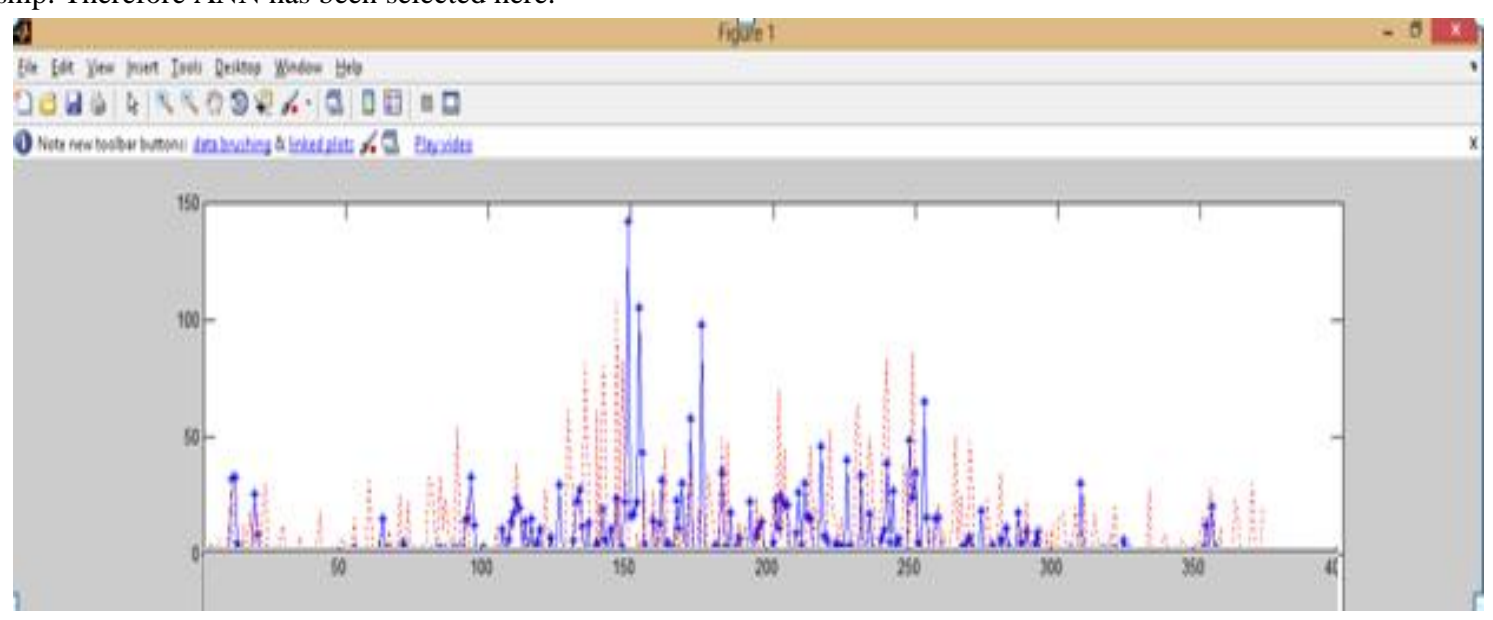

Figure 2: Predicted vs Actual output

Table-1

Performance Comparison for various Training Functions

Comparison between various most suitable Training functions in Back Propagation Network

\begin{tabular}{lllll}
\hline Type Of Network & \multicolumn{1}{c}{$\begin{array}{c}\text { Description of Training } \\
\text { pattern }\end{array}$} & $\begin{array}{c}\text { Training } \\
\text { Function }\end{array}$ & $\begin{array}{c}\text { Performance } \\
\text { Goal (MSE) }\end{array}$ & Regression \\
\hline $\begin{array}{l}\text { Condition1 } \\
\text { Feed Forward }\end{array}$ & Train LM & 0.31356 & $\mathrm{R}=0.93735$ \\
$\begin{array}{l}\text { Network(with fixed } \\
\text { No. of hidden layers) }\end{array}$ & $\begin{array}{l}(24,48,24) \\
\text { logsig tansig purelin }\end{array}$ & Train GDX & 0.31553 & $\mathrm{R}=0.93618$
\end{tabular}


$(24,24)$

Condition2

logsig tansig

Train LM

0.319

$\mathrm{R}=0.93634$

$(24,48,24)$

logsig tansig purelin

Train GDX

0.31989

$\mathrm{R}=0.93735$

$(24,50,24)$

tansig tansig purelin

Train GDA

0.3356

$\mathrm{R}=0.93878$

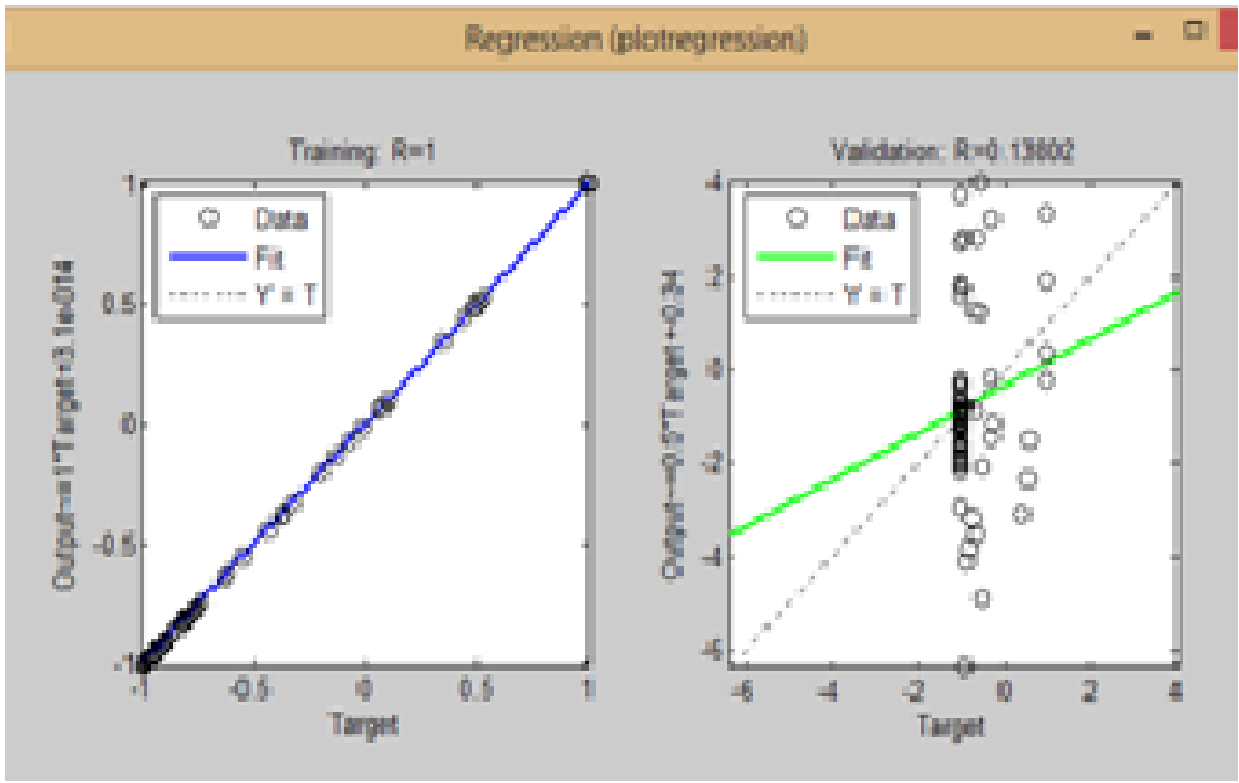

Figure 3: Post regression analysis on trainlm

\section{CONCLUSION}

Artificial neural network models were developed and applied for one day ahead each hour temperature prediction. The experimental results showed that a single hidden layer is sufficient to solve the non-linearity among the data variables. It is found that training algorithm is the most important factor in the performance and accuracy of the network. Post regression analysis and the comparison of output with the targets showed that if well organized training is conducted, the results attained are more accurate and precise.. Also the data to be taken into consideration significantly affects the training and output results.

Of all the training algorithms, LM proved to have minimum square error as well as correlation coefficient. these models exhibited better performance as compared to other statistical methods such as regression and correlation. They can achieve good learning performance because of their capability to learn non linear relationship between parameters taken. Also ANN's are able to generalize more as compared to other models. The modeling indicates that reasonable prediction accuracy was achieved for most of the models. Compared to regression models, the ANNs forecast the weather parameters with higher accuracy. The ANNs can be easily developed to perform multi-class classification problem without increasing the calculation complexity.

Proposed ANNs can be included for error detection and correction, learning linear and non linear equalization etc.
However, recent advancement in the design of computationally intelligent systems i.e feed forward time delay neural network may preferably be taken into consideration as it has shown promising results in other time series researches.

Sensitivity analysis or critical dependence of one parameter on others could be involved for the further study, as ANN models have played a significant role in forecasting the boundary conditions for the prediction of local climatic events.

\section{REFERENCES}

[1] Lorenz EN (1969) Three approaches to atmospheric predictability. Bull Am Meteorol Soc 50:345-349

[2] Kuligowski RJ, Barros AP (1998) localized precipitation forecasts from a numerical weather prediction model using ANNs. Weather forecast 13:1194-1205

[3] Maqsood I, Kan MR, Abraham A(2002a) intelligent weather monitoring systems using connectionist models. Neural parallel Sci Comput 10:157-178

[4] Maqsood I, Kan MR, Abraham A(2002b) neurocomputing based Canadian weather analysis. In Proceedings of the $2^{\text {nd }}$ international workshop on intelligent systems design and applications(ISDA'02), Atlanta, Georgia, August 2002. Dynamic Publishers, Atlanta, Georgia, pp 39-44 
[5] Guoqiang Zhang, B.Eddy Patuwo, Michael Y. Hu (1998) Forecasting with artificial neural networks: the state of the art. International journal of forecasting 14 (35-62), Elsevier

[6] Zurada JM(1992) introduction to artificial neural systems. West publishing company, saint paul, Minnesota

[7] Jimenez D, walsh $\mathrm{n}(1998)$ dynamically weighted ensemble neural networks for classification. In: proceedings of the international joint conference on neural networks(IJCNN 98), anchorage, Alaska, may 1998, pp 753-756

[8] Khan MR, ondrusek C (2000) short-term electric demand prognosis using artificial neural networks. Electr eng 51:296-300
[9] Wu, A., Hsieh, W.W. and Tang, B.(2006), Neural network forecasts of the tropical Pacific sea surface temperatures, Neural Networks, 19, 145-154.

[10] Nabia et al(2005), climate change prediction using data mining, ijicis, vol.5, no. 1 , july

[11] W.W Hsieh. and Tang B. (1998). Applying neural network models to prediction and data analysis in meteorology and oceanography. Bulletin of American Meteorological Society, Vol.79 1855-1870.

[12] Amr H El-Shafie, Hasan G El Mazoghi, A Shehata, Mohd R Taha.(2011). artificial neural network technique for rainfall forecasting applied to Alexandria, Egypt. International journ of physical science, vol(6)6, 13061316. 